\title{
Analytical Methodology to Evolutionary Steps for Manufacturing Strategy in Small and Medium Enterprises
}

\author{
Rricardo Palma ${ }^{1,}$ Laure $_{\text {Morel }}{ }^{2,}$ Raymundo Forradellas ${ }^{1}$ \\ ${ }^{1}$ Universidad Nacional de Cuyo, Facultad de Ingeniería, Maestría en Logística \\ Mendoza Argentina Centro Universitario (M5502KFA), Ciudad, Mendoza. Casilla \\ de Correo 405. República Argentina. \\ rpalma@uncu.edu.ar,kike@uncu.edu.ar \\ ${ }^{2}$ EquipeÉquipe de Recherche sur les Processus Innovatifs - Institute National \\ Politechnique de Lorraine - École Nationale Supérieure en Génie des Systèmes \\ Industriels ENSGSI, INPL - 8 rue Bastien Lepage B.P. 647 F54010 Nancy France \\ Laure.Morel@ensgsi.inpl-nancy.fr
}

\begin{abstract}
Usually, we can find lots of methods and recipes for operating in the field of strategy. Many distinguished and important organizations, like the United Nations, the Food and Agriculture Organization, the United Nations Industrial Development Organization, and others, work very hard to help a constellation of Small and Medium Enterprises (SME) across the world. These kinds of enterprises exist in the developed countries as well as in the emerging economies, but, usually, the global strategy that they adopt can't be easily synchronized with the manufacturing function. Then, it's not common to find in the literature an easy way to move the actual manufacturing strategy towards the goal proposed by the global strategy. In this work, an analytical methodology is proposed to align this manufacturing strategy with global strategies using as a motivating force the customer satisfaction, innovation and the systemic competitiveness framework. This approach is made in step way, making small evolutionary steps to convert the actual operation actions into a strong and ductile manufacturing strategy.
\end{abstract}

Keyword: Manufacturing Strategy, Innovation, Small and Medium Enterprise

\section{Introduction}

As part of the global strategy, manufacturing strategy contributes to the success or breakdown of an enterprise. It must be in harmony with marketing, financial and human resources, research, development, and innovation. If a company wants to be competitive, each part of his strategy must be something that is more than the sum of 
the isolated parts (synergy principle). No part is left out and no part dominates the other ones. Nevertheless, in some cases, business strategy is dominated by non manufacturing functions, with the result being "thrown over the wall" to manufacturing [11]. Manufacturing that is inadequate with strategic planning and tries to be all things to all people delivers mediocre performance. Nowadays, there is a growing interest in the SME around the world. As many authors point out, every big company has a root that leads to a brilliant idea developed in a garage. The gaps between those very different stages in the life of an enterprise are not only a matter of luck. Since Skinner [1] (1969) out lined the importance of a strategic alignment of the manufacturing function, manufacturing strategy has become one of the most discussed issues in the field of operations management. Many publications offer conceptual frameworks and give empirical evidence concerning the use of a manufacturing strategy. While extensive literature on manufacturing strategy has been written since the $1960 \mathrm{~s}$, still some research questions remain unanswered [2].Unfortunately, most of this effort is oriented to, or concerned with big companies or corporations and only a small part research effort is working on the problems of small and medium enterprises and how to align the manufacturing function with the global strategy, while contemplate at the same time the need to innovate in the SME. In this reduced set, only five recent works have been found, thus, this subject's data includes the manufacturing strategy as a key factor on the company's innovation process. One of the strengths of the small and medium enterprises is their capacity for high innovation in products; however, the tools and soft technology that apply to solve the problem of innovate in the manufacturing system are not concerned with the (or are part of) the global strategy. Usually this problem is see as a dichotomy mater where the SME must to decide between innovate or align the manufacturing strategy with the global strategy, but not the same things at the same time.

\section{Scope}

Many research programs say that the scope of the manufacturing strategy concept is not totally clear [3]. It's well known that the objective of the manufacturing strategy is to get a more competitive enterprise and help match the goals of the global strategy. More recent literature exposes the impact of the shortened life cycle, and additional studies state that the manufacturing has become transformed in some kind of cryptic thing. Lots of anachronisms composed by three letter words like EOQ (Economic Order Quantity), JIT (Just In Time), SCM (Supply Chain Management), and CRM (Customer Relationship Management) appear to make more difficult to understand what a manufacturing strategy implies. Then the manager confuses "manufacturing strategy" with "manufacturing executive technology" [4]. The solutions proposed by the many researchers and other organizations can help enterprises, but not in every aspect, since the methodology proposed is the same to all different types of companies. Thus, the help of an expert is needed to customize the solutions for each special case. In this work, a methodology is proposed but the final result is different for each company. The methodology here proposed assumes a need to evaluate the competitive status of the management team and the manufacturing responsibility on 
it. At the same time, the presence of a consultant is not needed to implement the manufacturing strategy (a diy ${ }^{*}$ approach). The methodology is based in the fact that each change (evolution) in the product or service that the company brings necessarily must be followed by a change in the manufacturing strategy [5] (co-evolutions) and, consequently, a proper design of the supply network is needed to grant the stability and flexibility of the system (adaptation). In this case the evolution force is the innovation process that pushes the company and its manufacturing strategy to adapt itself to the market's needs. The methodology offers at the same time an additional value to the stake holders that let him to see and understand more clearly the change in the environment (local and global) where the company operates (lives).

\section{Hypotheses}

3.1 It is possible to improve the global strategy of the company using some metric that limits the field of feasible solutions adopted by the manufacturing strategy. This improvement can be defined as the ability of the company and its manufacturing system to change the manufactured product by an innovative one, and the process to satisfy the ever shortest life cycle imposed by the customer to the kind of product manufactured.

3.2 Using the metrics that limit the field of decisions of the manufacturing strategy, it's possible to isolate a collection of manufacturing policies that can improve the alignment manufacturing strategy with the corporate strategy. At the same time, a better performance in the supply chain that leads to a low level inventory and a high flexibility may be obtained. This process has been observed in some clusters of high competitive companies where the innovation is extensively applied.

\section{Research work and development of the methodology}

\subsection{Summary}

To develop the method proposed in this work, a three phases approach (with several steps each one) has been followed. In the first phase, extensive bibliography and articles' reading has been made. Additionally to this reading, a preliminary diagnostic has been made over 175 enterprises using the metrics obtained in the literature revision.

In this diagnostic, only 49 companies have said to believe that the manufacturing strategy impacts in the innovation process. Thus, the research has worked only with this sub set of companies that have completed a preliminary survey. As a consequence of the small number in the sample, is not possible to obtain a high level of confidence.

${ }^{*}$ DIY (Do It Yourself) 
But this reduced set of sample is enough to work in the region where to research program operates. However, an approach to the use a discrete events simulation techniques [15] can be used to improve the confidence level. This simulation can be implemented in the third phase as a corrective factor. In this simulation is possible to find two copy of each company that uses the same manufacturing strategy.

By comparing many of the successful manufacturing strategies and best practices cited by the literature with the successful ones found in the set of companies, a list of key factors was isolated using PROMETHEE analysis method [6]. This method uses as input the manufacturing strategy (alternatives) and the attitude that the company has faced to the innovation (criteria) to rank the manufacturing strategy. Finally a ranking of "fittest" manufacturing strategy is obtained. At the same time a classification criteria is given to us by the method to understand which group of company can be able to integrate a cluster, because they uses the same criteria, and how important is the innovation in his success ( to see more detail read Morel et al.[11] and [6].

At this point strategy adopted by each company is exposed. Nevertheless, the non relevant information, such as the customer soft selection criteria, best and worst partner in the supply chain and so forth, is stored in a knowledge base, mixed with relevant one. This is made in this way because some irrelevant factors may become critical in the future or in subsequent scenarios. The final result of the first phase is displayed as a rating table where the manufacturing practices are sorted from the most to the least important. This is the result of the first phase, and is itself useful for the company. It will be used as feeds for the second phase.

The second phase explores the potential capacity of the existing manufacturing strategy to cover the gap presented between actual and desired operating level. To decide on the proper step to be taken, a mixed methodology of data mining and human decision is used. Initially, the methodology offers to the decision taker (a human being) a collection of sequential sets of actions to be taken that are compatible with the constraints that the company has (especially financial constraints). Each time that the methodology sees a confirmation form of the human actor, a weight factor is increased for the rule that brings this conclusion.

If the human actor rejects the step, then the weight factor is decreased for the reasoning process that arrives to this conclusion. Then, in a second enterprise that uses the methodology, the ranking of suggested step is affected by the experience increased by the previous interaction. Using this interaction the method is trying to work as a "substitute" of the consultant. As it will be explained later, a treatment supported by data mining techniques is used. To this research a modified variation of the C 4.5 [19] algorithm is used. This algorithm can build decision trees and isolate cluster of consistent step to cover the gap, but C 4.5 algorithm can't use verbal judgments and only can use numerical judgments. So a modification had been made, as the reader can see over there. C 4.5 is a well known basic tool used to learn the basis of data mining and can be used under GNU license.

Finally, in the third phase, using as entry the confirmed step form the phase 2, a suggested, a sequence of alignment of the production resources is constructed with 
special attention to Human Resources, Organizational Structure, Marketing, Technological Management, Process Management, Product and Material Technology and Financial Infrastructure. When the improvement is observed in the global strategy, the information is fed back to the knowledge base to improve the methodology of the Phase 2 and 3 (double feedback). This step is not totally developed at the current moment and it has only had a successful experience in the wine industry and textile cluster in the region of Mendoza, República Argentina. Then the reader can see only experimentation in such industry leaving the rest of the activities to a future work.

\subsection{The three phases methodology}

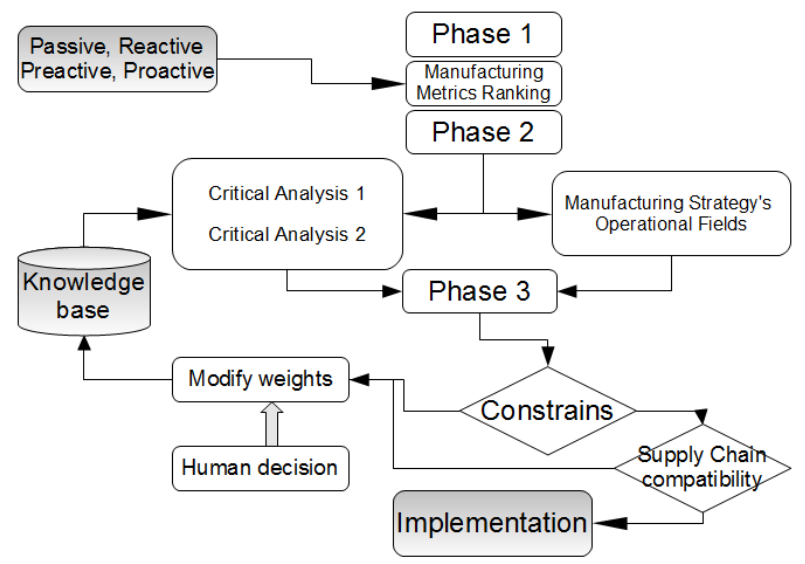

Fig. 1. Resume map of the methodology. The figure shows the steps that the methodology follows to adapt the manufacturing strategy to the customer needs and supply chain existing structure while try to innovate in the process.

\subsubsection{The First Phase}

The proposed methodology has 3 phases. The first one is basically a diagnosis about the health care of the company among its manufacturing system and the capability to synchronize it with the supply chain (pulled by the client). As a result, we have at this 
point a list of desired actions to improve the innovation and adapt the company and the manufacturing strategy to the market's needs.

A collection of frameworks and methods from the literature have been analyzed. Amongst others, the reader can see Miller and Roth [8] that think about this context in the same way as adopted by this work. Then, manufacturing strategy consists of two core elements: the manufacturing task, and the pattern of manufacturing choices.

First, we should know what the manufacturing function must accomplish [9]. Skinner exposed this concept as "the competitive priorities" such as quality, cost, delivery or flexibility.

Second, we should be concerned with the major decisions on manufacturing structure and infrastructure that a company needs to achieve his goal, it's addressed by manufacturing tasks and his possible weaknesses [10]. But in the SME field this metrics can become less important because the customers demand other things linked to the product. The customer likes fast customer services or tailored solutions that the big companies can't offer. This fact is usually observed in the software factory company or textile industry (in the sub set of company under study). However, this particularity of the SME is assumed as true for the rest of the company. Other metric that the customer appreciates in the SME is the innovation capacity. For the small company this metric may be the difference between life and death.

To develop this diagnostic first phase the emphasis must be put in this metric.

A deeper search in the literature can help us filter only methods and metrics that point to innovation as a key factor to the small enterprises. Supporting this idea, an approach is proposed, based on a work proposed by [11] and [6]. A numerical assessment that qualifies the innovative attitude of the enterprise is adopted.

Table1. Verbal scale used in the phase 1

\begin{tabular}{c|c} 
Attitude & Numeric Scale \\
\hline Proactive & 0.75 to 1 \\
Preactive & 0.50 to 0.75 \\
Reactive & 0.25 to 0.50 \\
Pasive & 0 to 0.25
\end{tabular}

The four words used to describe the innovation attitude of the enterprise are based in a recommendation used by Godet [13] and also used by Morel [6] To make the performance evaluation of phase 1 , a collection of manufacturing indicators is proposed and a comparison between the market and the supply chain where the company work is made to develop the PROMETHEE ranking (see table 2). To make the survey of this phase, a small form has been developed using Google Docs; and the company fill the form, can compare his performance with the mean of the region as a "Balanced Score Card" [20] (see table 2).

Any company can fill in the "Google Form" to make the performance survey. Accordingly, the Supplier and the Customer (the nearest supply chain echelons) are compared in term of "Passive, Reactive, Preactive or Proactive" against the SME. 
This is made by a numerical judgment. Afterwards, the company is informed about the main competitor (if the information is available at the knowledge base). Finally, the company must choose the strategy adopted by each metric (maximize o minimize). If the company says that the preference is to maximize flexibility, it means that the company wants to be (at least) the near the best in the market. This does not necessarily mean that the company wants to be proactive in the flexibility criteria of the manufacturing process. In this example, all the weight factors of the PROMETHE method [11] have been set to the same value, but using the third phase each individual weight can be moved to make successive improvement to the methodology.

Table2. Ranking obtained by the phase 1. This example show the result obtained for one of companies that works in the textile sector in the region. All the companies works with skins, combined with cotton or wool combined in a clothing, then the flexibility of the manufacturing the most important in the ranking.

\begin{tabular}{l|rr} 
Manufacturing metric & Rank (importance) & \\
\hline Flexibility & I \\
Manufacturing Cost & II \\
Logistics SCM & II \\
Innovation & IV \\
Quality & V \\
Manufacturing Cost & VI & \\
$\ldots$ & $\ldots$ & \\
$\ldots$ & $\ldots$ & \\
Inventory Level & XXXII most important
\end{tabular}

\subsubsection{The Second Phase}

This phase uses as a start point the ranking build with PROMETHE and comparing it to the strengths and weaknesses of the actual production policy. As a result, we can obtain a list of possible ways to evolve (change) the manufacturing strategy to a high operating level that can satisfy the customer's needs and, at the same time, satisfy the constraints imposed by the company like capacity, budget, machinery, and so on. The actions to take are offered by the methodology using a knowledge base, but human decision is also needed as was previously informed, especially in the first instances of use when the knowledge base is still empty. Once the decision has been made, the third step establishes the competitive factor's importance, such as quality, cost, delivery on time, success index and flexibility and others like manufacturing technology, facilities and infrastructure permeability to incorporate new innovative product [3]. As result, a second ranking is made and compared with the company's real capacity to achieve these requirements [4]. One additional thing that can be obtained at this point is the direction that the changing factor must follow. (for example is not absolutely necessary ever to minimize the operating cost to get a better performance.) 
Table 3. Company Performance compared against de main actors in the supply chain and in the market.

Manufacturing process metrics

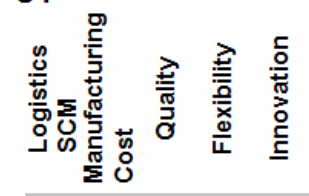

\begin{tabular}{l|l|l|l|l|l|l|}
\multicolumn{1}{l|}{ Max / Min } & Max Min Max Max Max \\
\hline Supplier & 0,77 & 0,92 & 0,22 & 0,52 & 0,24 \\
\hline Our Company & $\mathbf{0 , 7 5}$ & $\mathbf{0 , 7 6}$ & $\mathbf{0 . 2 2}$ & $\mathbf{0 , 5 5}$ & $\mathbf{0 , 8 5}$ \\
\hline Customer & 0,43 & 0,65 & 0.22 & 0,51 & 0,61 \\
\hline $\begin{array}{l}\text { Mean in the } \\
\text { market }\end{array}$ & 0,65 & 0,78 & 0.25 & 0,53 & 0,72 \\
\hline $\begin{array}{l}\text { Best in the mar- } \\
\text { ket }\end{array}$ & 0,78 & 0.55 & 0.35 & 0,55 & 0,9 \\
\hline $\begin{array}{l}\text { Main Competit- } \\
\text { or }\end{array}$ & 0,66 & 0.55 & 0,28 & 0.34 & 0,6 \\
\hline
\end{tabular}

Note 1. Information showed to the user (Table 3) of the google web form showing his enterprise performance. The user only must to load the row marked as "Our Company"

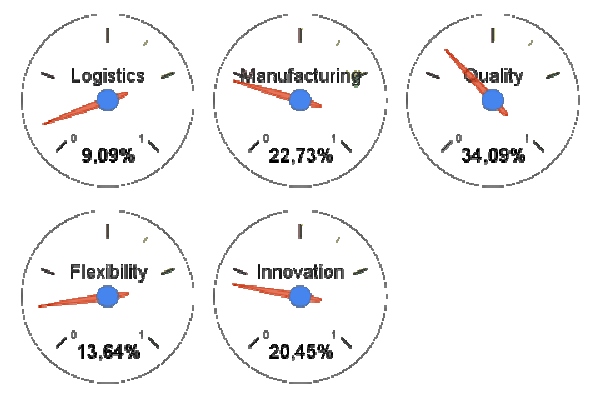

Fig. 2. Result offered to the company. Implemented over Google technology API the performance can be observed. The table 3 is converted in the figure 2

\section{Volumes / Products Critical Analysis}

In the phase 2 two critical analysis must be done. The first critical analysis that is made compares the actual and desired performances obtained from the first step. Basically, a comparison between (I) Logistics and SCM, (II) Manufacturing Cost, (III) Quality, (IV) Flexibility and (V) Innovation Capabilities is confronted with the usually adopted policies like: 
a) Manufacture one product in a super high volume

b) Some products, high volume c) Few products, medium volume

d) Many products, small volume

e) Many products, individual units.

Then, a question must be answered thinking about the impact the change of actual manufacturing policy (among "a" to " $\mathrm{e}$ ") in the performance of the metric may have (I to VI). For example, if the first phase is showing that the company is at the "reactive level" in the quality metric, and according to the maximize criteria adopted to this metric, the PROMETHEE ranking says that this metric is a priority; we must see if the actual manufacturing policy, say for example, type "e" (that means craftsman work); can be moved to "d" (small lot or batch processing).

This process is implemented as a dialogue in a consultant help or Google Form where the decision taker is sequentially asked for each combination pairs of policy/metrics. The human response can take numeric values from 0 to 1 . If there is a potential contribution when some policy is adopted, it contributes "1" (or does not contribute " 0 " ) to move quality attitude from Passive to Reactive.

When the change in the manufacturing strategy is made and the new product delivered to the market, a final diagnosis is made to feed back data to the knowledge base and increase the reliability and viability of the methodology's actions suggested in step two.

\section{Product Flow / Layout Critical Analysis}

The second critical analysis uses the same metrics provided by the PROMETHEE ranking, which are confronted against the followings policies :

a) Functional Layout - Intermittent and varied fow

b) Cell Layout - Regular Flow with patterns

c) Linear Layout - (Equip or machine line) Continuous fow

d) Linear Layout (Buildings line) Uninterrupted Streaming

Similar to the previous critical analysis, a confrontation between "a to d" policy is made against the "I to VI" metrics. Following the previous example, assume that the ranking is telling us that the "Manufacturing Cost" must be moved from reactive to proactive level, according to the best competitor in the market.

Then, the decision taker is asked by the impact to move the actual layout / product flow to a new one in the Manufacturing cost.

Although in both analyses questions are answered in values from 0 to 1 , nothing prevents the study to provide trial answers involving numbers that represent units, money, time or inventory. It's necessary though that the responses are dimensionally homogeneous throughout the interrogation. 


\section{Selecting the proper manufacturing (soft) technology}

It's possible to see the result of the dialogue between the human expert and the questions proposed by the critical analysis in table 3 .

Using the response of the expert, it can be possible to know which metric receives more impact than others when we change the two analyzed policies. It's also possible to determine a decreasing order of impact importance using the Principal Component Analysis (PCA), but more comprehensible results can be obtained using the J48 [17] algorithm ( a modification of classical C 4.5 classification tree algorithm [19]).

Table 4. Typical report observed in the textile cluster companies

Here, ( Table 4 and 5) we can see the result of the overall impact consequence. If the impact in the innovation capability metric is less or equal to $45 \%$ and the quality metric is less than $8 \%$ then the overall impact expected will be low (less than $20 \%$ ). If quality is greater than $8 \%$ then the innovation capability is better than $1 \%$ and the overall impact will be Medium (70\%), and so on.

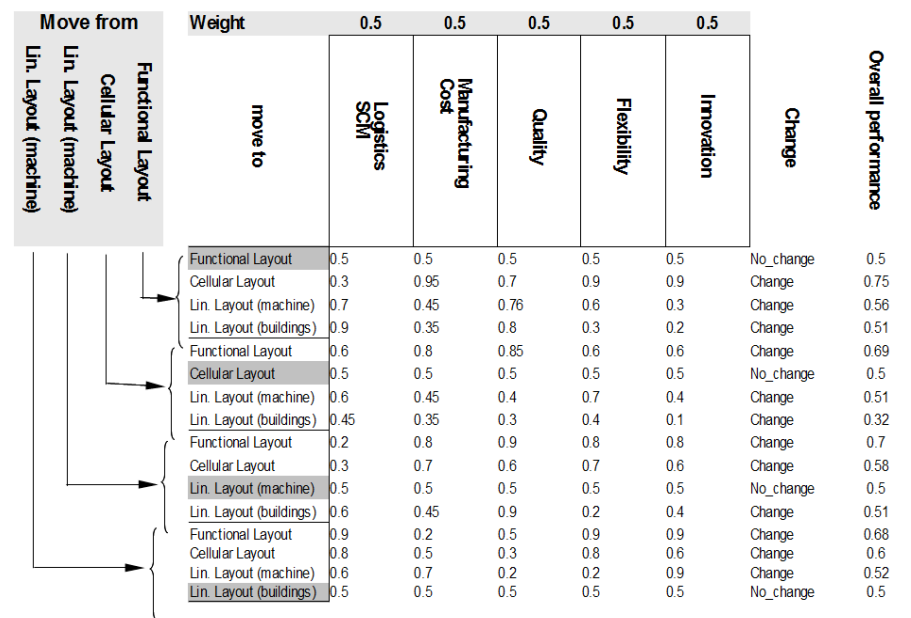

Table 5. Result of the tree classification technique J48 pruned tree for the overall impact in textile

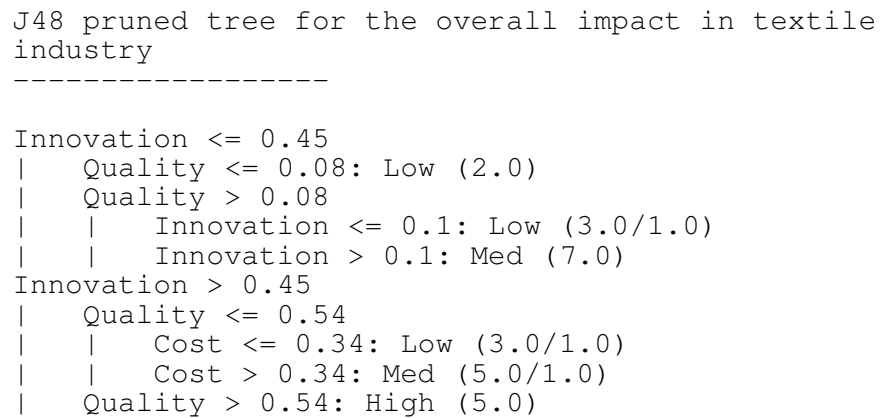


The same reasoning can be interpreted using a tree graphic as shown in figure 2 .

Since C4.5 algorithm can handle numeric attributes, then a modification introduced by University of Waikato [14] is made to the original algorithm to work with the judgment (Low, Medium and High), this translation is not necessarily linear. The use of human decision feedback can alter the linearity of this algorithm. This new variant is totally parametric in Weka [14] and is called J48 algorithm and has been used in this work.

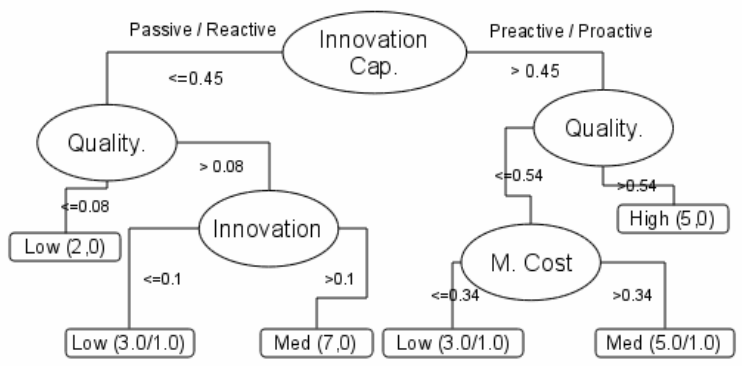

Figure 3 Knowledge tree of the critical analysis for textile cluster

It is clear now that the knowledge rule constructed by J48 algorithm is showing that the Innovation Capabilities of the manufacturing process are the most important metric for this kind of enterprises.

Then follows that the Innovation followed by Quality and after the Manufacturing Cost are the key factors that impact in the global strategy and depend on the manufacturing strategy.

It is possible to continue to the moment when all the metrics are used, but in the initial condition, to operate the J48 algorithm, a confidence interval of $86 \%$ has been used. As a consequence, only with three metrics is possible to explain all the variability of this behavior. The final result is the same that can be obtained using de PCA method. At the bottom line it is possible to observe that 25 rectangles (result of $2+3+7+3+5+5$ ) instances have been classified (see figure 3). Only three instances show a degree of inexactitude that is identified as 3.0/0.1. That means that 3 instances are correctly classified with an $86 \%$ of confidence and 1 instance is not.

Using the same technique, it is possible to operate in the second critical analysis (flow / layout). In table 5, a summary of results is showed.

In table 5, two additional rows are showed. These rows remark where the manufacturing policy is impacted by the layout and the material flow. Then it is possible to observe "changed" or "not changed" and the overall impact in the performance (in a range of a numeric range from 0 to 1 .

Table 6. Typical response found in the form filled by wine industry 


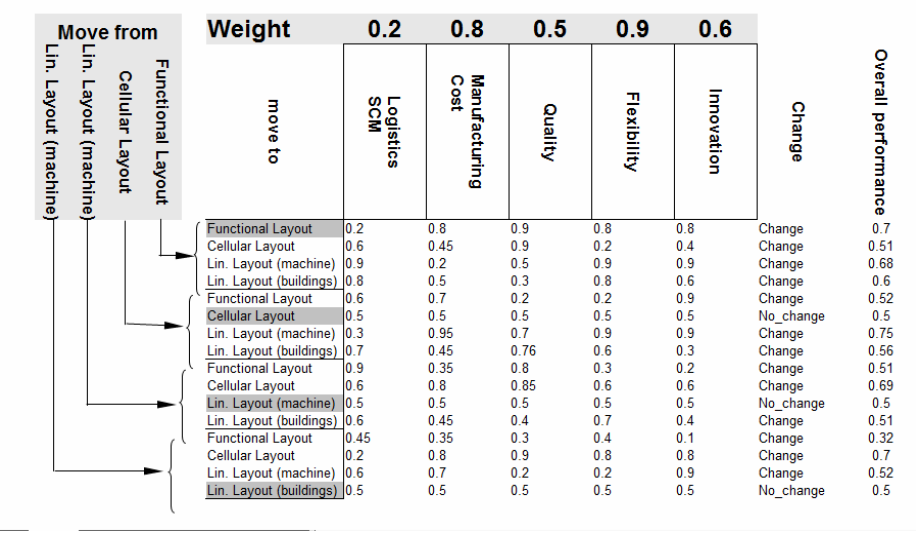

Attention must be take on row at the top of table 6 shows the weight factors that had been moved by the feedback process of the third phase.

Table 7. A more detailed report of the wine industry classification tree J48 pruned tree for the overall impact in wine industry

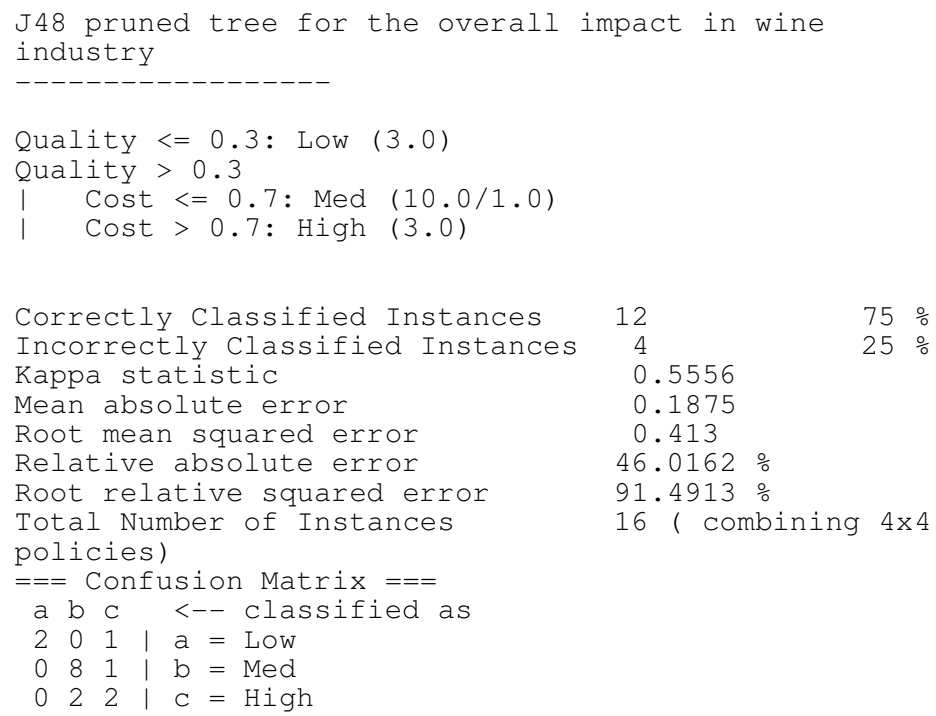

Here (Table 7) we can see that when the impact of the layout and material flow is used to measure the impact in the five metrics, only the Quality and Cost are significant. With only two variables, it is possible to explain the behavior of the change in the policy established in the second critical analysis showed in the Product Flow / Layout Critical Analysis. 


\section{Cross validation of the two critical analyses}

Using the two critical analyses, it is possible to establish the field of the most effective manufacturing strategy, comparing the layout / product volume policy according to [3] and [12] .

To establish which manufacturing strategy must be used, it is necessary to find in table 3 the actual manufacturing policy. ( One Product/High Volume , Few product / High Volume, Some Products / Med. Vol. , Many Products / Small Vol. , Many Products / Individual Units). Then we must choose the policy where we want to move. To make the decision, we must read the impact only in the column "Innovation Capability" and "Quality", because they are the metrics that explain nearly all the behavior in textile cluster. The combination that gives us the best global performance must be chosen. If no difference in the instances is observed, then the Manufacturing Cost must be considered. These three metrics are the most significant for the textile industry in the region used by the survey.

As a result of the last work we can state that "Some Products / Med Vol" and "Many Products / Medium Volume" are the best manufacturing strategy for this case.

Repeating the last process with table 7, it is possible to isolate the second manufacturing strategy that has high impact in the metric. Then, we suppose that for the textile cluster, the work leads us to the election of "Cellular Layout" and "Lineal Layout (machine oriented)". Observing the place where the two policies interact, we can see that "Batch Flow", "Operation Placed Line Flow" and "Lean JIT" manufacturing strategies are the best choice to obtain the desired operating level discovered in the first phase.

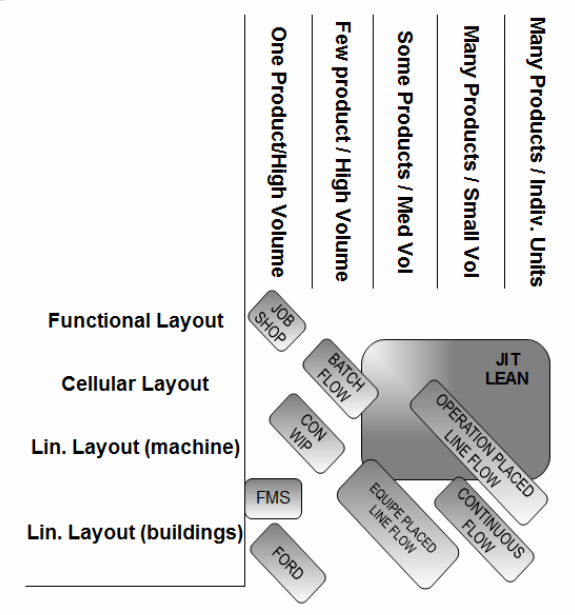

Figure 4 operational fields for each manufacturing strategy. According to the type of layout and the products manufactured is possible to choose the manufacturing strategy 


\section{Construction of the third phase}

This phase use the data collected from the companies that have implemented the change in the manufacturing strategy and uses the information to decrease the uncertainty of the methodology. This is an ever converging process [16]. Each time that e constraint is violated the feedback process try to change the weight (see table 6). It is possible to make better recommendations using the new column in the data used in table 7 to adapt the J48 algorithm (overall change column). The performance of each change in the decision rule reduces the covariance of the matrix constructed by the metrics of the first phase and the policies exposed in the second phase. In the same way, the use of the weight factor in table 3 and 4 is always a convergent process [2]. This process can offer a more simple way to improve the methodology if the sequence of actions and decisions follow the collaborative optimization technique suggested by [18].

This feedback process continuously affects the J48 algorithm's result and so far no evidence has been found that the process tends to diverge or oscillate.

This phase is not yet implemented as a web service, but it will be possible in the future to develop it. At this moment is implemented as a excel prototype.

Each time that a strategy manufacturing field is proposed this option must be checked to see if the constraints, that the company has, can be met. If they can't be met, then the weight used in the phase 2 must be changed using the action suggested by an expert or choose by a company that have similar weights. In a second instance, the compatibility with the supply chain must be tested. The company must usually ask itself whether its storage capacity, lead time and production rate are able to work with the supplier and customer capacity. Also the own capacity and infrastructure must be checked

If the supply chain is not satisfied, then the weight used in the phase 2 must be increased. This modification is registered in the knowledge base.

\section{Results and final discussion}

As was previously exposed, only 49 companies have followed the survey during 2009/2010.

From the total population than initially began the survey (175), 30 companies that have not followed the survey have found the bankruptcy. At this moment, we don't have more information about this part of the population. On other hand, from the set of 49 studied companies only 2 have gone bankrupt. In the sample set of 49 companies, it is possible to find many activities (see table 8).

As a consequence of the reduced number of samples, the confidence level was not useful for the case where we have only one company. (Material's quality, Electromechanical and Renewable Energy). 
In olive oil, we have a special case because this is a single company but it has 7 different locations placed near the different cultivated (crop) zones. Then the confidence level can be improved in a new work that is being developed.

The initial confidence level as a means for the experiment is near $86 \%$ and could be improved if the process is repeated every year (especially if we use extensively the third phase).

As a consequence of the second phase, the initial hypotheses 3.1 have been proved. To prove the second conjecture (3.2) a discrete event simulation model is proposed. To make the simulation, software [15] has been used.

Table 8. Detailed lists of the company sectors that have participated in the survey, and final decision about the Hypotheses 1 and 2 expressed by the 49 companies

\begin{tabular}{l|r|c|c} 
Sector & Cant. & Hypotheses 1 & Hypotheses 2 \\
\hline Aeronautic & 2 & & Verify \\
Agri food & 3 & Verify & Verify \\
Communication & 2 & Verify & \\
Building & 6 & Verify & \\
Materials Quality & 1 & & Verify \\
Electromechanics & 1 & Verify & \\
Energy & 1 & & Verify \\
Industrial Gases & 3 & Verify & \\
Logistics & 2 & & Verify \\
Wood & 2 & Verify & \\
Metal Mechanics & 7 & & Verify \\
Olive Oil & 1 & Verify & \\
Industrial Senvices & 4 & & \\
Textile & 5 & Verify & Verify \\
Wine & 9 & Verify & Verify
\end{tabular}

\section{References}

1 - Skiner, Wickham - 1969 - p38 Manufacturing, the missing link in corporate from Production and operations management (Spanish version) - Ed. Wiley

2 - Jörn-Henrik, Thun - 2008 - Empirical analysis of manufacturing strategy implementation, International Journal of Production Economics, 113, issue 1, p. 370382.

3- Swamidass P., Newell W. - 1997- Manufacturing strategy, environmental uncertainty, and performance : A path analytic model, Management Science 33 (1987) 509-524.

4 - Boo-Ho Rho , Kwangtae Park, Yung-Mok Yu - 2001 - An international comparison of the election of manufacturing strategy implementation gap on business performance [B1D] Int. J. Production Economics 70 (2001) p 89 to97 
5 - Emmanuel D. Adamidesa, Nikolaos Pomonisa - The co-evolution of product, production and supply chain decisions, and the emergence of manufacturing strategy Int. J. Production Economics 121 (2009) p 301-312.

6 - Morel, Nemery and Camargo, 2009 A metrics-based diagnosis tool for enhancing innovation capabilities in SMEs, Conference for the Workshop on Multicriteria decision analysis for enhancing innovation capabilities in SMEs, ERPI laboratory, 2-3 fevrier 2009 Nancy France.

7 - Godet, Michel - 1990 - Integration of scenarios and strategic management : Using relevant, consistent and likely scenarios, Futures, Volume 22, Is- sue 7, September 1990, Pages 730-739, ISSN 0016-3287, DOI : 10.1016/0016- 3287(90)90029-H.

8 - Miller J.C. , Roth A.V. , A taxonomy of manufacturing strategies, Management Science 40 (3) (1994) pp 285-304.

9 - Skinner W. , Manufacturing in the Corporate Strategy, Wiley, New York, 1998 REVIWED EDITION.

10 - Hayes R.H. , Wheelwright S.C. - Restoring our competitive edge: competing through manufacturing, Wiley, New York, 1984.

11 - Jean-Pierre Brans and Bertrand Mareschal, How to Decide with PROMETHEE (GAIA Software) ULB and VUB Brussels Universities - Vrije Universiteit Brussel. http://homepages.ulb.ac.be/ bmaresc/PromWeb.htm

12 - Miltenburg, John - Manufacturing Strategy 2nd edition. ISBN 978-1563273179

13 - Dunod, E. Gestion Industrielle, Paris, France, 1997

14 - Waikato University Weka Machine Learning Framework http://www.cs.waikato.ac.nz/ml/weka

15 - Simul 8 Software, a simulation workbench to discrete event process. www.simul8.com

16 - Wallace Hopp and Mark Spearman Factory Physics 2nd Ed. ISBN 9780071232463

17 - Witten I.H. and Frank E. Data Mining : Practical Machine Learning Tools and Techniques (Second Edition) ISBN 0-12-088407-0.

18 - De Miguel E. Adida - Supply Chain Competition with Multiple Manufacturers and Retailers, Forthcoming in Operations Research, (2010) http://faculty.london.edu/avmiguel/papers.html

19 - Ross Quinlan C 4.5 algorithm, http://en.wikipedia.org/wiki/Ross_Quinlan\#C4.5 20 - Kaplan R., Norton D : Harvard Business Review (1996) Volume: 29, Issue: February 1996, Publisher: Harvard Business School Press, Pages: 4-9 ISSN: 01900528 ISBN: 0875846513 